\title{
An Analysis on Finding the Influencing Factors of Supporting for the "GiveitUp" LPG Subsidy for the Government using Data Mining Techniques
}

\author{
K. Chitra Lekha \\ M.Phil(CS) Research Scholar, \\ Department of Computer \\ Science and Applications \\ SCSVMV University, Enathur \\ Kanchipuram.
}

\author{
S. Prakasam, M.C.A, PhD \\ Associate Professor, \\ Department of Computer \\ Science and Applications \\ SCSVMV University, Enathur, \\ Kanchipuram.
}

\begin{abstract}
The Liquefied Petroleum Gas (LPG) is light distillate, uncontaminated and efficient form of energy obtained from crude oil and the processing of natural gas. The main objective of this work is to find the respondents who support LPG subsidy "GiveitUp" scheme for the welfare of the Indian government. The purpose of this work is to investigate the obstacles that stand in the way of not surrendering the LPG subsidy. A survey has been conducted during January-April 2016 with different category respondents of 276. The questionnaire was designed to predict the factors about "GiveitUp" LPG subsidy scheme among the various sectors respondents of the society. The respondents' survey was distributed from face to face contact in and around Kanchipuram. The WEKA software is used for the study implementation since it contains a collection of visualization tools and algorithms for data analysis. In this dissertation author used PredictiveApriori algorithm as it generates ' $n$ ' best association rules based on $\mathrm{n}$ selected and Classification and Regression Tree (CART) that predict categorical class labels. Ranking gives the binary judgement among various attributes. The SPSS tool is used to Cross tabulate between the respondents with higher income and their opinion of surrendering LPG subsidy and also between the respondents living in rural areas and their opinion of preventing the withdrawal of LPG subsidy for the government.
\end{abstract}

\section{Keywords}

WEKA, Data Mining, PredictiveApriori, CART.

\section{INTRODUCTION}

Among all the subsidies that are provided by the Indian government, one of the subsidies that reach directly to the families Below Poverty Line (BPL) is LPG subsidy. The main aim of LPG subsidy scheme was to light up the rural homes, helping the weaker section of the society. The Indian government launched Direct Benefit Transfer or DBT on January 1, 2013 to change the process of transferring subsidies[19]. The DBT transfers subsidies directly to the people through their bank account. Leakages, delays, corruptions etc will be reduced by crediting subsidies in to bank accounts. Under LPG subsidy, all households are entitled to get 12 cylinders of LPG, each with $14.2 \mathrm{~kg}$ at a subsidized rate of Rs.419.26, against the market price of Rs.608.

The NDA government requested the government officials and professionals of the country to give up their LPG subscription believing that it would help many mothers and households of our country. According to the NDA government, every LPG consumer who surrender cooking gas subsidy is linked to a
BPL household that gets the LPG connection in turn. As on December 7 2015, according to Ministry statistics, a total of $52,58,841$ consumers have given up or surrendered their subsidy. Taking into account the quota of a cylinder per consumer and average LPG subsidy of Rs.184.93/cylinder (in Delhi market) for the period from April to September,2015,the annual saving would be approximately Rs.1167crore. Dumka in Jharkhand has become the first place where 5000 households from BPL category were handover LPG connections by Modi on October 2, 2015. A survey has been conducted during January-April 2016 with respondents of various categories of 276. The WEKA software is used in this study for prediction techniques that predict the respondent's opinion about withdrawal of LPG subsidy for the benefits of government. In WEKA ChisquaredAttributeEval evaluates the worth of an attribute by computing the value of the chisquared statistic with respect to the class. In this dissertation author used PredictiveApriori technique as it searches with an increasing support threshold for the best ' $n$ ' rules concerning a support based corrected confidence value. This analysis identifies the benefits for the government if the respondents surrender their LPG subsidy. The some of major factors are : Decrease government's subsidy expenditure bill, Help weaker section, At least one BPL gets LPG connection, Reduce indoor pollution, Light up rural homes, Lower fiscal deficit, Stabilize economy. The PredictiveApriori analysis shows the result obtained is benefits for government supporting factor is to light up rural homes and respondents' preventing factor of not giving LPG subsidy is that respondents wants to utilize the subsidies that are provided by the government. Ranking gives a numerical or ordinal score to the attributes our ranking score is 322.763 to One BPL gets LPG connection and 351.185 to utilize the subsidies that are provided by the government.

\section{LITERATURE REVIEW}

Nan Jiang and Le Gruenwald (2012) emerging applications of data streams that require association rule mining, such as network traffic monitoring and web click streams analysis. Different from data in traditional static databases, data streams typically arrive continuously in high speed with huge amount and changing data distribution [15]. This raises new issues that need to be considered when developing association rule mining techniques for stream data. They discuss those issues and how they are addressed in the existing literature. Generate exact mining results by maintaining a small subset of frequent item sets from data streams and keeping their exact frequency counts [Yang, 2004, Chi, 2004, Mao,2005]. To keep track of the exact frequency counts of target item sets with limited memory space, one way is to adopt the sliding window data 
processing model, which maintains only part of the frequent item sets in sliding window(s) as in[Chi, 2004].

Divya Bansal \& Lekha Bhambhu (2013) are saying that Apriori is to find frequent item sets and to uncover the hidden information. Apriori Algorithm is used to discover and understand the underlying patterns involved in the court's records from their data contains in various sections [11].

Stefan Mutter , Mark Hall \& Eibe Frank (2013) Pruning a rule set output by predictive Apriori takes less time than pruning one output by Apriori, because predictive Apriori prunes away many rules during the search using its inherent pruning strategy.

Paresh Tanna \& Dr. Yogesh Ghodasara(2014) are saying that how the different approaches achieve the objective of frequent mining along with the complexities required to perform the job. The purpose of reducing the number of scans of database to extract frequent item set will be resolved in future due to our work is in progress for the same[9].

Jyothsna R. Nayak and Diane J. Cook (2014) are saying that an associate rule mining algorithm that searches for approximate association rules. Our $\sim$ AR approach allows data that approximately matches the pattern to contribute toward the overall support of the pattern. This approach is also useful in processing missing data, which probabilistically contributes to the support of possibly matching patterns. Results of the $\sim \mathrm{AR}$ algorithm are demonstrated using the Weka system and sample databases. It demonstrates the effectiveness of the $\sim$ AR algorithm using sample databases. Results indicate that $\sim$ AR successfully generates rules that approximate true correlations in the input database. This behavior is beneficial for databases with many missing values or containing numeric data.

Hetal Thakkar, Barzan Mozafari and Carlo Zaniolo (2014) The real-time (or just-on-time) requirement associated with online association rule mining implies the need to expedite the analysis and validation of the many candidate rules, which are typically created from the discovered frequent patterns. Moreover, the mining process, from data cleaning to post-mining, can no longer be structured as a sequence of steps performed by the analyst, but must be streamlined into a workflow supported by an efficient system providing quality of service guarantees that are expected from modern Data Stream Management Systems (DSMSs).

Pratiyush Guleria \& Manu Sood(2015) Predictive Apriori Algorithm uses pruning strategy where it searches for the best rules and highly accurate rules which are included by less accurate ones remain part of the output. Predictive Apriori uses an increasing support values and is able to mine a high quality set of association rules. Predictive Apriori algorithm performs well when it is used to generate a small set of rules.

K.Raghavendra Swamy, G.Harinadh Babu \& R.Venkatasubbaiah (2015) are saying that market basket analysis used for to identify the most associated items, and this type of analysis used for the Business improvement and introduction of new products and study the different transactions, how the different transactions are carried out and how the business needs are changed. By using Apriori algorithm to generate new List items and Candidate keys, depending on the Confidence Factors.

Tobias Scheffer (2015) when evaluating association rules, rules that differ in both support and confidence have to be compared; a larger support has to be traded against a higher confidence.

Jatinder Kaur \& Jasmeet Singh Gurm(2015) are saying that goal of data mining process is to extract information from a data set and transform it in to an understandable structure for further use. There are many classification algorithms but CART builds both classification and regression trees.

Surjeet Kumar Yadhav \& Saurabh Pal(2012), Educational data mining is used to study the data available in the educational field and bring out the hidden knowledge from it. The C4.5,ID3 and CART decision tree algorithm predict the performance of the students. The comparative analysis of the results states that the prediction has helped the weaker students to improve and brought out betterment in the result.

\section{METHODOLOGY}

The data mining technique based on analysis finding factor that support the withdrawal of LPG subsidy for the government using PredictiveApriori is carried out in this work. The CART algorithm is used to predict the most influencing factor that support and prevent the withdrawal of LPG subsidy by the respondents for the government. In this work, the respondents earning annual income from 5 to more than 10 lakhs are categorized as respondents with high income. The respondents earning annual income from 5 lakhs to less than 1 lakh are categorized as respondents with low income. The data analysis helps in better understanding of large set of data.

\subsection{PredictiveApriori Algorithm in WEKA}

The PredictiveApriori algorithm generates Apriori association rules using predictive accuracy. The predictive accuracy is the combination of support and confidence values. In WEKA software, select the Explorer in Applications panel in WEKA GUI Chooser and open WEKA Explorer. Open the saved .csv file and convert it in to .arff file and then preprocess the file. Then select the Associate tab and click Choose button. From associator models, choose PredictiveApriori algorithm and click the start button to run the algorithm. Thus the best rules for association of factors supporting the withdrawal of LPG subsidy for the government by the respondents are generated using PredictiveApriori algorithm.

\subsection{Classification and Regression Tree (CART) in WEKA}

Classification And Regression Tree (CART) was introduced by Breiman in 1984 . It builds both classification and regression trees. Based on binary splitting of the attributes, Classification and Regression tree is built. CART is used for data exploration and prediction also. CART is non parametric, therefore this method does not require specification of any functional form. CART algorithm itself identifies the most significant variables and eliminate non-significant variables.

Classification Trees: where the target variable is categorical and the tree is used to identify the "class" with in which a target variable would likely fall in to.

Regression Trees: where the target variable is continuous and tree is used to predict it's value.

To know the efficiency of prediction system, CART algorithm has been used.

\subsection{Cross Tabulation using SPSS}

SPSS is capable of handling large amounts of data and can perform all of the statistical analyses. The SPSS GUI has two 
views namely Data view and Variable view which can be toggled by clicking on the tab in the bottom of the left in SPSS window. The Data view shows the spread sheet view of the cases (rows) and the variable view shows variables(columns). The variable view displays the metadata dictionary each row represents a variable and shows variable name, variable label, measurement type and variety of other characteristics.

\section{DATA ANALYSIS}

The questionnaire has been designed to measure the effectiveness of "Giveit Up" LPG subsidy scheme among the various respondents in and around Kanchipuram. The purpose of this work are : (i) To find whether higher income respondents are willing to give LPG subsidy. (ii) To find the reasons why respondents living in rural area are not accepting the withdrawal of LPG subsidy. This paper presents how the collected data are analyzed through appropriate data mining techniques and the results of data analysis.

\subsection{Population and sample}

The author collected 276 samples from the data among which there are 111 male respondents and 165 female respondents.

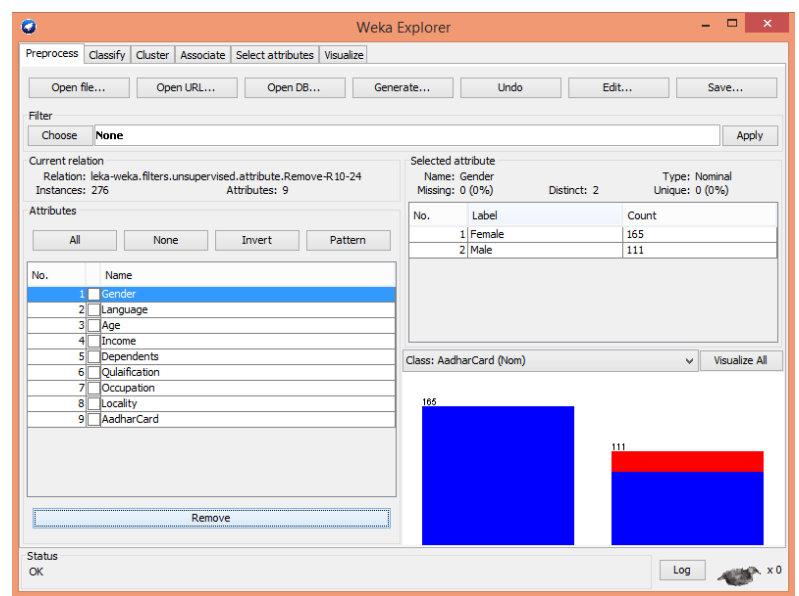

Fig 4.1Screenshot for Sample in WEKA Explorer

\subsection{Hypothesis tested}

\subsubsection{Research Hypothesis (HI)}

There is an association between the higher income respondents and their willingness to surrender LPG subsidy for the Indian government.

\section{Null Hypothesis (H0)}

There is no association between the higher income respondents and their willingness to surrender LPG subsidy for Indian government.

The total number of instances used for association is 276 . The attributes used for generating best rules for PredictiveApriori algorithm are Decrease subsidy expenditure bill, Help weaker section, One BPL gets LPG connection, Strengthen agriculture sector, Reduce indoor pollution, Light up the rural homes, Lower fiscal deficit, Stabilize the economy based upon the attribute family annual income. The full training set is used as Associator model. The result of PredictiveApriori algorithm is shown in fig 4.2

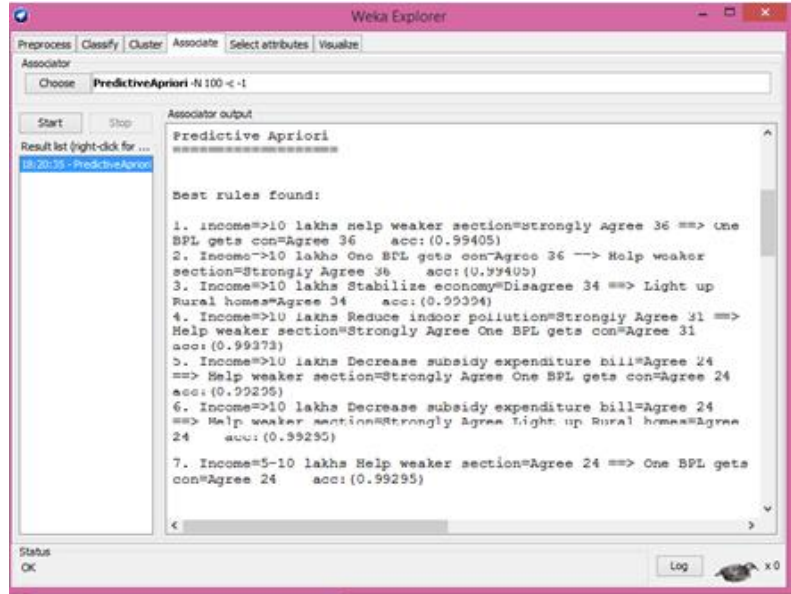

Fig 4.2 Screenshot for Higher income respondents are willing to give LPG subsidy.

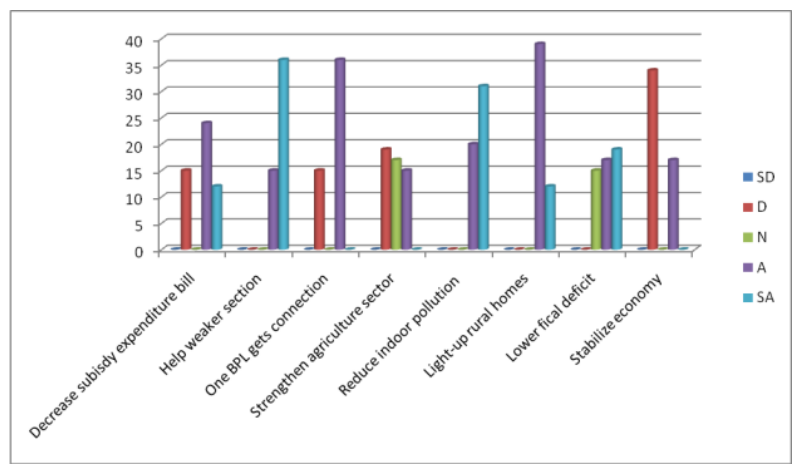

Fig 4.3 Higher income respondents are willing to give LPG subsidy.

Here output for the given is clearly see the much influencing factors are Light up rural homes and One BPL gets connection. These two are the highly influencing factors which support withdrawal of LPG subsidy among higher income respondents. We associated these factor by the attribute family annual income.

\subsubsection{Research Hypothesis (H1)}

There is an association between the respondents living in rural areas and their opinion of not surrendering the LPG subsidy for the Indian government.

\section{Null Hypothesis (H0)}

There is no association between the respondents living in rural areas and their opinion of not surrendering the LPG subsidy for the Indian government.

The total number of instances used for association is 276 . The attributes used for generating best rules for PredictiveApriori algorithm are Believe it as their rights, Corruption less scheme, Utilize best of its benefits, Is government transparent of PAHAL scheme, Previous method is comfortable, Not ready to pay full amount, Public infrastructure development based upon the attribute Area of locality. The full training set is used as Associator model. The result of PredictiveApriori algorithm is shown in fig 4.4 


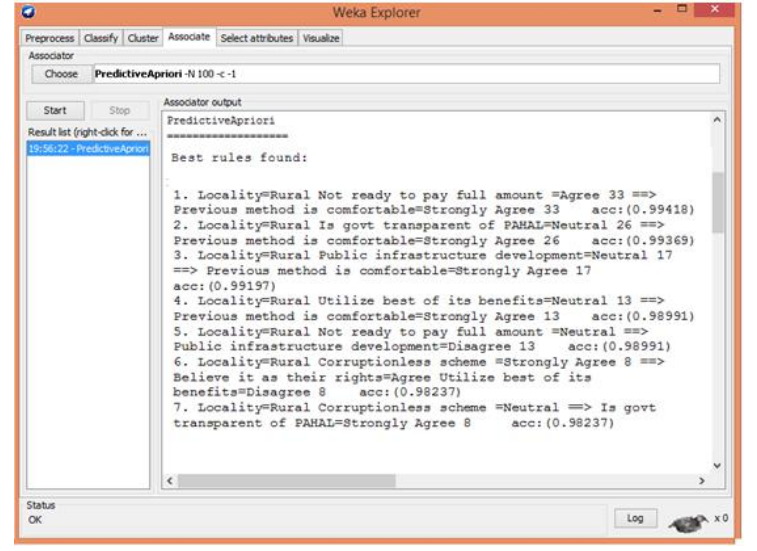

Fig 4.4 Screenshot for respondents living in Rural area are not willing to give LPG subsidy.

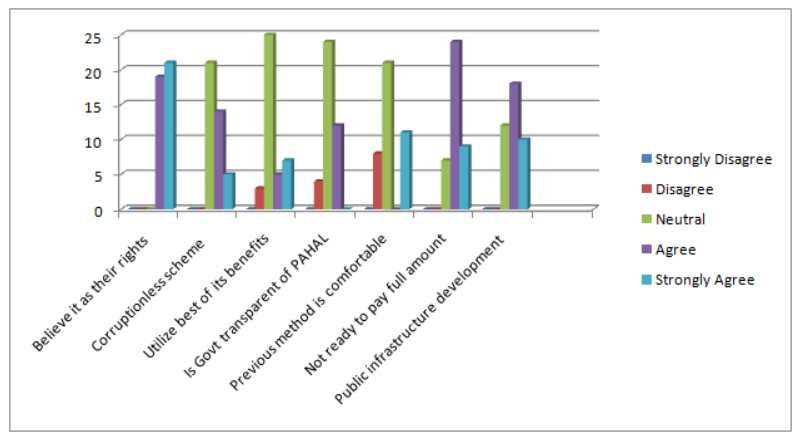

Fig 4.5 Respondents living in rural area are not willing to give LPG subsidy.

Here output for the given is clearly see the much influencing factors are Not ready to pay full amount and Believe it as their rights. These three are the highly influencing factors which prevent surrendering of LPG subsidy among the respondents living in rural areas. We associated these factors by the attribute Area of locality.

\subsection{Classification and Regression Tree (CART) in WEKA}

CART builds multivariate decision (binary) trees that is available in WEKA as SimpleCART. It is best suited for training data. To build a tree, CART algorithm uses Pruning method. The data has been classified recursively until it has been categorized as perfectly as possible in CART algorithm.. The confusion matrix showed the factors supporting the withdrawal of LPG subsidy by the respondents. Here the prediction is done by the attributes Decrease subsidy expenditure bill, Help weaker section, One BPL gets LPG connection, Strengthen agriculture sector, Reduce indoor pollution, Light up rural homes, Lower fiscal deficit, Stabilize economy and the predicted income result is described below. The decision tree is created based on which classification on the test data is done. In our study, confusion matrix (contingency table) has five classes, and so a $5 * 5$ confusion matrix. The sum of diagonals in the matrix is the number of correctly classified instances, all others are incorrectly classified instances.

$===$ Confusion Matrix $===$

a b c d e <-- classified as

69 \begin{tabular}{llll|l}
6 & 0 & 0 & 0 & $\mathrm{a}=5-10$ lakhs
\end{tabular}

\begin{tabular}{llll|l}
188 & 2 & 0 & 0 & $\mathrm{~b}=<1$ lakh
\end{tabular}
\begin{tabular}{llll|l}
1282 & 0 & 0 & $\mathrm{c}=3-5$ & lakhs
\end{tabular}
\begin{tabular}{lllll|l}
0 & 0 & 0 & 9 & 0 & $\mathrm{~d}=>10$ lakhs
\end{tabular}
\begin{tabular}{lllll|l}
5 & 2 & 5 & 1 & 9 & $\mathrm{e}=1-3$ \\
\end{tabular}

The correctly classified instances are $257(93.1159 \%)$ which is the sum of diagonals of confusion matrix $(69+88+82+9+9)$ and the incorrectly classified instances are 19(6.8841\%).

Table 1: Prediction based on respondents' annual income

\begin{tabular}{|c|c|c|c|c|c|c|c|}
\hline \multirow[b]{2}{*}{$\mathrm{O}$} & & \multicolumn{5}{|c|}{ Predicted } & \multirow{2}{*}{$\begin{array}{c}\% \text { of } \\
\text { correctly } \\
\text { predicted }\end{array}$} \\
\hline & & $\mathrm{a}$ & $\mathrm{b}$ & $\mathrm{c}$ & $\bar{d}$ & $\mathrm{E}$ & \\
\hline $\begin{array}{l}\mathrm{b} \\
\mathrm{s}\end{array}$ & 5-10lakhs & $\begin{array}{l}69 \\
(69)\end{array}$ & 0 & 0 & 0 & 0 & 100 \\
\hline $\mathrm{e}$ & $<11$ lakh & 1 & $\begin{array}{l}88 \\
(91)\end{array}$ & 2 & 0 & 0 & 93.40 \\
\hline $\mathrm{v}$ & 3-5lakhs & 1 & 2 & $\begin{array}{l}82 \\
(85)\end{array}$ & 0 & 0 & 96.47 \\
\hline d & $>$ 10lakhs & 0 & 0 & 0 & $9(9)$ & 0 & 100 \\
\hline & 1-3lakhs & 5 & 2 & 5 & 1 & $\begin{array}{l}9 \\
(22)\end{array}$ & 40.90 \\
\hline
\end{tabular}

Among 257 correctly classified instances, 100\% of respondents earning annual income between 5 to 10 lakhs disagree; $93.40 \%$ of respondents earning annual income less than 1 lakh agree; $96.47 \%$ of respondents earning annual income between 3 to 5 lakhs neutral; $100 \%$ of respondents earning annual income more than 10 lakhs strongly disagree and $40.90 \%$ of respondents earning annual income between 1 to 3 lakhs strongly agree.

Table 2: One BPL gets LPG connection Vs Income

\begin{tabular}{|c|c|c|c|c|c|c|c|}
\hline \multirow{2}{*}{\multicolumn{2}{|c|}{$\begin{array}{l}\text { One BPL gets LPG } \\
\text { connection Vs } \\
\text { Income }\end{array}$}} & \multicolumn{5}{|c|}{ Income (in lakhs) } & \multirow[t]{2}{*}{ Total } \\
\hline & & \multirow{2}{*}{$\begin{array}{l}<1 \\
0\end{array}$} & \multirow{2}{*}{$\begin{array}{l}1-3 \\
0\end{array}$} & \multirow{2}{*}{$\begin{array}{l}3-5 \\
14\end{array}$} & \multirow{2}{*}{$\begin{array}{l}5-10 \\
10\end{array}$} & \multirow{2}{*}{$\begin{array}{l}>10 \\
0\end{array}$} & \\
\hline $\begin{array}{l}\text { One } \\
\text { BPL }\end{array}$ & $\begin{array}{l}\text { Strongly } \\
\text { Disagree }\end{array}$ & & & & & & 24 \\
\hline gets & Disagree & 0 & 0 & 9 & 0 & 15 & 24 \\
\hline LPG & Neutral & 0 & 24 & 4 & 0 & 0 & 28 \\
\hline connect & Agree & 26 & 24 & 56 & 24 & 36 & 166 \\
\hline ion & $\begin{array}{l}\text { Strongly } \\
\text { Agree }\end{array}$ & 14 & 1 & 19 & 0 & 0 & 34 \\
\hline Total & & 40 & 49 & 102 & 34 & 51 & 276 \\
\hline
\end{tabular}

From the above cross tabulation, respondents' main reason for supporting withdrawal of LPG for the government is One Below Poverty Line(BPL) gets LPG connection in turn. Among 40 respondents with annual income earning less than 1 lakh have high influencing percent 26 of them agree. Among 49 respondents with annual income earning between 1to3 lakhs have high influencing percent 24 of them are neutral. Among 102 respondents with annual income earning between 3 to5 lakhs have high influencing percent 56 of them agree. Among 34 respondents with annual income earning between 5 to10 lakhs have high influencing percent 24 of them agree. Among 51 respondents with annual income more than 10 lakhs have high influencing percent 36 of them agree. The above tabulation concludes that most of the respondents agree to withdraw LPG subsidy for the government so that One Below Poverty Line(BPL) gets LPG connection. 
Table 3: Outcome of study Income Vs One BPL gets LPG connection

\begin{tabular}{|c|c|c|}
\hline Income & Description & $\begin{array}{c}\text { Outcome of } \\
\text { study }\end{array}$ \\
\hline \multirow{2}{*}{$\begin{array}{c}\text { One BPL gets } \\
\text { LPG connection }\end{array}$} & $\begin{array}{c}\text { To find respondent } \\
\text { earning high or low } \\
\text { income(1-10 lakhs) }\end{array}$ & Positive \\
\cline { 2 - 3 } & $\begin{array}{c}\text { High income } \\
\text { respondents(5 to above } \\
10 \text { lakhs })\end{array}$ & Positive \\
\cline { 2 - 3 } & $\begin{array}{c}\text { Low income } \\
\text { respondents(3 to below } \\
1 \text { lakh) }\end{array}$ & Negative \\
\hline
\end{tabular}

Table 4: Believe it as their rights Vs Income

\begin{tabular}{|c|c|c|c|c|c|c|c|}
\hline \multirow{2}{*}{\multicolumn{2}{|c|}{$\begin{array}{l}\text { Believe it as their } \\
\text { rights Vs Income }\end{array}$}} & \multicolumn{5}{|c|}{ Income (in lakhs) } & \multirow[t]{2}{*}{ Total } \\
\hline & & $<1$ & $1-3$ & $3-5$ & $5-10$ & $>10$ & \\
\hline \multirow{5}{*}{$\begin{array}{l}\text { Believe } \\
\text { it as } \\
\text { their } \\
\text { rights }\end{array}$} & $\begin{array}{l}\text { Strongly } \\
\text { Disagree }\end{array}$ & 0 & 0 & 0 & 8 & 0 & 8 \\
\hline & Disagree & 0 & 0 & 5 & 0 & 0 & 5 \\
\hline & Neutral & 0 & 18 & 16 & 11 & 0 & 45 \\
\hline & Agree & 19 & 3 & 54 & 12 & 36 & 124 \\
\hline & $\begin{array}{l}\text { Strongly } \\
\text { Agree }\end{array}$ & 21 & 28 & 27 & 3 & 15 & 94 \\
\hline \multicolumn{2}{|l|}{ Total } & 40 & 49 & 102 & 34 & 51 & 276 \\
\hline
\end{tabular}

From the above cross tabulation, respondents' main reason for preventing the withdrawing of LPG subsidy for the government is that the respondents believe that the subsidies that are provided by the government are their birth rights and they want to utilize them properly. Among 40 respondents earning annual income less than 1 lakh have high influencing percent 21 of them strongly agree. Among 49 respondents earning annual income between 1 to 3 lakhs have high influencing percent 28 of them strongly agree. Among 102 respondents earning 3 to 5 lakhs have high influencing percent 54 of them agree. Among 34 respondents earning annual income between 5 to 10 lakhs have high influencing percent 12 of them agree. Among 51 respondents earning annual income more than 10 lakhs have high influencing percent 36 of them agree.

Table 5: Outcome of study Income Vs Believe it as their rights

\begin{tabular}{|c|c|c|}
\hline \multirow{2}{*}{ Income } & $\begin{array}{c}\text { Description } \\
\text { To find respondent } \\
\text { earning high or low } \\
\text { income(1-10 lakhs) }\end{array}$ & $\begin{array}{c}\text { Outcome of } \\
\text { study }\end{array}$ \\
\hline \multirow{2}{*}{$\begin{array}{c}\text { Believe it as } \\
\text { their rights }\end{array}$} & $\begin{array}{c}\text { High income } \\
\text { respondents(5 to above } \\
10 \text { lakhs) }\end{array}$ & Negative \\
\cline { 2 - 3 } & $\begin{array}{c}\text { Low income } \\
\text { respondents(3 to below } \\
1 \text { lakh) }\end{array}$ & Positive \\
\hline
\end{tabular}

\subsection{Chi-squared attribute evaluator for ranking}

In this study, for ranking Chi-squared attribute evaluator method is chosen and Ranker is selected as search method. Full training set is used as attribute selection mode.

\subsubsection{Ranking based on respondents' annual income}

The attributes used for Chi-squared attribute evaluator for ranking are Decrease subsidy expenditure bill, Help weaker section, One BPL gets LPG connection, Strengthen agriculture sector, Reduce indoor pollution, Light up the rural homes, Lower fiscal deficit, Stabilize the economy based upon the attribute family annual income.

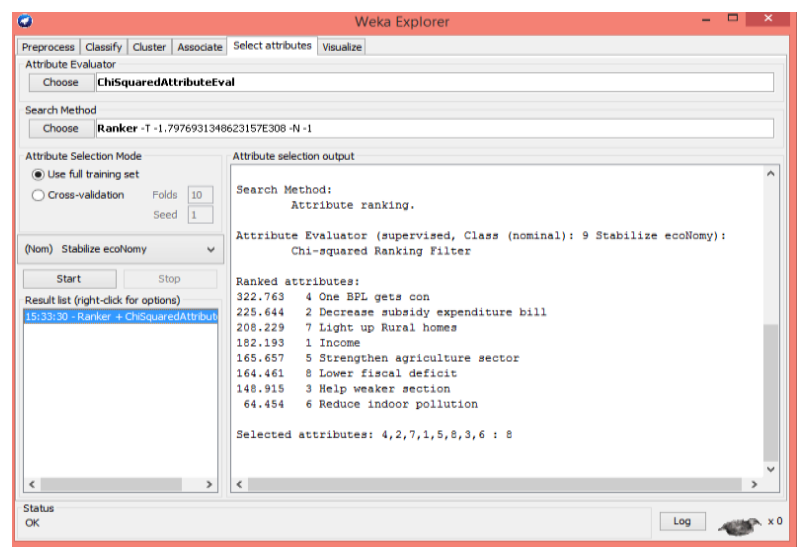

Fig.4.6. Screenshot for ranking among the opinion of respondents based on their annual income

The ranking algorithm is used to find the most influencing factor of the large set of data. Here output percentage for the given is clearly see the much influencing factors are One BPL gets LPG connection322.763 and Decrease subsidy expenditure bill225.644. These two are the highly influencing factor which supports withdrawal of LPG subsidy among respondents. We ranked these factors by the attribute family annual income [10].

\subsubsection{Ranking based on respondents' area of locality}

The attributes used for Chi-squared attribute evaluator for ranking are Believe it as their rights, Corruption less scheme, Utilize best of its benefits, Is government transparent of PAHAL scheme, Previous method is comfortable, Not ready to pay full amount, Public infrastructure development based upon the attribute Area of locality.[10]

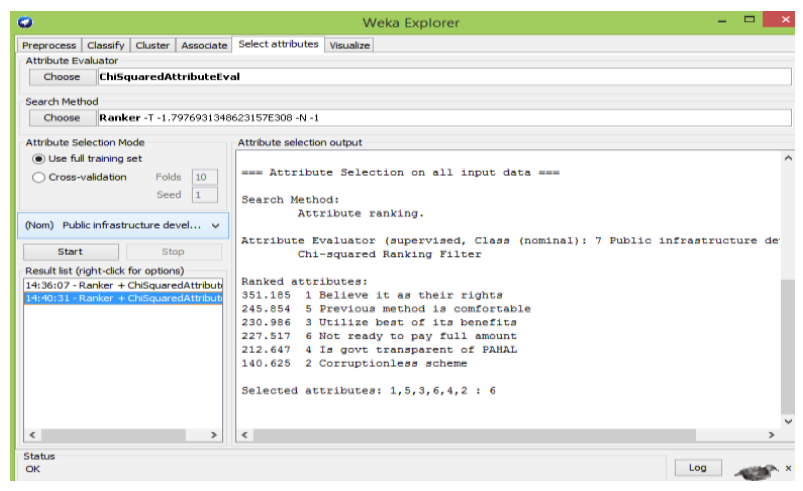

Fig.4.7: Screenshot for ranking among the opinion of respondents based on their area of locality. 
Here output percentage for the given is clearly see the much influencing factors are Believe it as their rights 351.185 and previous method is comfortable245.854. These two are highly influencing factor which prevent the withdrawal of LPG subsidy among the respondents. We ranked these factor by the attribute area of locality.

\section{CONCLUSION}

The LPG subsidy scheme will enable the LPG customers to get subsidy directly to their respective bank accounts. The WEKA software is used for the study implementation since it contains a collection of visualization tools and algorithms for data analysis. In this dissertation author used PredictiveApriori technique as it identifies the benefits for the government if the respondents surrender their LPG subsidy. The some of major factors are : Decrease government's subsidy expenditure bill, Help weaker section, Atleast one BPL gets LPG connection, Reduce indoor pollution, Light up rural homes, Lower fiscal deficit, Stabilize economy. The PredictiveApriori analysis shows the result obtained is benefits for government supporting factor is to light up rural homes, One BPL gets LPG connection and respondents' preventing factor of not giving LPG subsidy is utilize the subsidies provided by the government. Our prediction analysis has been categorized in two different views based on respondents' annual income. The most influencing factor supporting the withdrawing of LPG subsidy among the respondents earning annual income from 5 to more than 10 lakhs is One BPL gets LPG connection and preventing factor among respondents earning annual income from 3 to less than 1 lakh is Believe it as their rights. Ranking gives a numerical or ordinal score to the attributes our ranking score is 322.763 to One BPL gets LPG connection where ranking is based on attribute family annual income. Ranking score is 351.185 to believe it as their rights where ranking is based on attribute Area of locality.

\section{REFERENCES}

[1] Akansha Sanjay Jain and Nitish Sunil Patil Crude oil prices and its impact on Indian economy .International Journal of Social and Humanties Research Vol.3,Issue 2,(562-572),April-June 2015.

[2] M.Prema and S.Prakasam Effectiveness of Data Mining based E-learning system (DMBELS). International Journal of Computer Applications (0975-8887) Volume 66-No.19,March 2013.

[3] Moawia Elfaki Yahia and M.Urtada El-mukashfi El-taher A new approach for Evaluation of Data mining Techniques.International Journal of Computer Science Issues, Vol.7,Issue 5, September 2010.

[4] Dr.Lokanatha C.Reddy, A Revie on Data mining from Past to Future, International Journal of Computer Applications(0975 - 8887) Volume 15- No.7, February 2011.

[5] MoPNG.(2013c).LPG Cooking Fuel:A Quantum Leap in Service to the customers.PIB. Retrieved from http://pib.nic.in/newsite/efeatures.aspx?relid=98430.
[6] MoPNG(2013d).One time grant to BPL Families for availing New LPG Connections under RGGLVY. Retrieved November 04, 2014,from http://pib.nic.in/newsite/erelease. aspx?relid=95136

[7] MoPNG.(2014c).LPG Connections to BPL Families.Retrieved November 04,2014,from http://pib.nic.in/newsite/PrintRelease.aspx?relid =106563

[8] Paresh Tanna and Dr.Yogesh Ghodasara Using APRIORI with WEKA for frequent Pattern Mining .International Journal of Engineering Trends and Technology(IJETT)Volume 12 No.3 - June 2014

[9] S.Dhivya and S.Prakasam An analysis on finding influencing factor of preventing the employment among the educated women using data mining techniques. International Journal of Computer Applications (0975 8887) Volume 101- No. 7. September 2014.

[10] K. Raghavendera Swamy and G. Harinadh babu and R. Venkata Subbaiah Identification of Frequent item search Patterns using APRIORI algorithm and WEKA Tool. International Journal of Innovative Technology and Research Volume No.3, Issue No.5, August - September 2015, 2401-2403.

[11] Divya Bansal and Lekha Bhambhu Execution of APRIORI algorithm of Data mining Directed towards Tumultuous crimes concerning Women . International Journal of Advanced Research in Computer science and Software Engineering Volume 3,Issue 9, September 2013

[12] Ms.Shweta and Dr.Kanwal Garg Miniing Efficient Association rules through APRIORI algorithms. International Journal of Advanced Research in Computer science and Software Engineering. Volume 3, Issue 6, June 2013.

[13] Shruti Aggarwal and Ranveer Kaur, Comparative Study of various Improved versions of APRIORI Algorithm

[14] Lok Sabha.(2013).LPG DISTRIBUTION NETWORK IN RURAL AREAS. Retrived July15, 2014, from http://164.100.47.132/Annexture_New/Isq15/11/au930.ht $\mathrm{ml}$.

[15] Nan Jiang and Le Gruenwald(2012),Research Issues in Data Stream Association Rule Mining,SIGMOD Record, Vol. 35, No. 1, March.2006

[16] Jatinder Kaur and Jasmeet Singh Gurm Description of Genetic and CART Algorithm using Data Mining Tool, International Journal of Advanced Research in Computer Science and Software Engineering Volume 5, Issue 6, June 2015.

[17] Surjeet Kumar Yadhav and Saurabh Pal, Data Mining : A Prediction for Performance Improvement of Engineering students using Classification, World of Computer Science and Information Technology Journal Volume 2,No.2,51-56, 2012

[18] http://www.giveitup.in

[19] http://www.pahal.ac.in 